\title{
Reply: Placenta previa and perinatal mortality
}

\author{
Gali Pariente $\cdot$ Eyal Sheiner
}

Received: 13 July 2011 / Accepted: 16 July 2011 / Published online: 4 August 2011

(C) Springer-Verlag 2011

\section{Dear Editor,}

We thank Dr Matsubara for the interest in our study. Our study demonstrated an increased risk of perinatal mortality by 5.6 times among patients with placenta previa. However, while using a multivariable logistic regression model, with perinatal mortality as the outcome variable, controlling for confounders, such as preterm birth, maternal age, intra uterine growth restriction (IUGR), etc., placenta previa was not found as an independent risk factor for perinatal mortality [1].

The present analysis, which surely has its inherent limitations, is, to the best of our knowledge, one of the largest analyses reported to date considering placenta previa and perinatal mortality. This analysis does support an increase in perinatal mortality among pregnancies with placenta previa in comparison with those without it. Nevertheless, it seems that the mortality is not attributed to the previa per se, but rather to the associated risk factors. Moreover, similar to the results of the current study, a previous study performed in our medical center found higher rates of perinatal mortality in pregnancies complicated with placenta previa [2]. However, while using multivariable analysis investigating risk factors for perinatal mortality, placenta previa per se was actually found as a protective factor against the occurrence of perinatal mortality while controlling for confounders.

There are many examples over the history of medicine in general, and obstetrics in particular, of beliefs that have been based on assumptions, concerns and personal impression rather than on facts and evidence based medicine. We cautiously attempt otherwise. Whether it is the previa per se or not, this condition, undoubtedly, is significantly associated with adverse perinatal outcome, and accordingly careful surveillance is recommended with timely delivery in order to reduce the associated complications.

Conflict of interest No conflict of interest exists.

\section{References}

1. Rosenberg T, Pariente G, Sergienko R, Wiznitzer A, Sheiner E (2011) Critical analysis of risk factors and outcome of placenta previa. Arch Gynecol Obstet 284(1):47-51

2. Sheiner E, Shoham-Vardi I, Hallak M, Hershkowitz R, Katz M, Mazor M (2001) Placenta previa: obstetric risk factors, pregnancy outcome. J Matern Fetal Med 10(6):414-419
G. Pariente $\cdot$ E. Sheiner $(\bowtie)$

Department of Obstetrics and Gynecology, Soroka University

Medical Center, Ben-Gurion University of the Negev,

P.O Box 151, Be'er Sheva, Israel

e-mail: sheiner@bgu.ac.il 